\title{
An HST search for planets in the lower main sequence of the globular cluster NGC $6397^{\star}$
}

\author{
V. Nascimbeni ${ }^{1,2,3, \star \star}$, L. R. Bedin ${ }^{2,3}$, G. Piotto ${ }^{1,2}$, F. De Marchi ${ }^{4}$, and R. M. Rich ${ }^{5}$
}

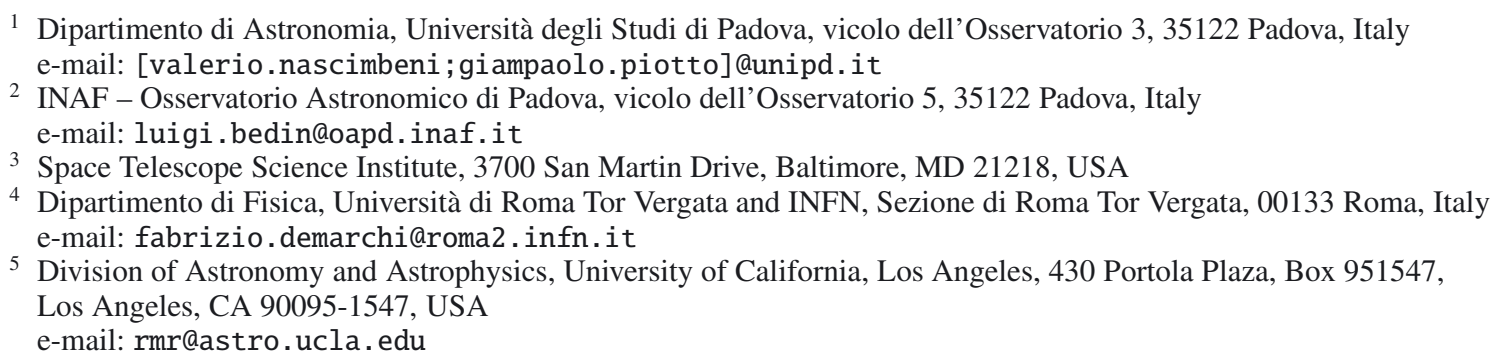

Received 15 December 2011 / Accepted 16 February 2012

\begin{abstract}
Searches for planetary transits carried out in open and globular clusters have to date yielded only a handful of weak, unconfirmed candidates. These results have been interpreted as either being insignificant, or evidence that the cluster chemical or dynamical environment inhibits planetary formation or survival. Most campaigns have been limited by small sample statistics or systematics from ground-based photometry. We performed a search for transiting planets and variables in a deep stellar field of NGC 6397 imaged by HST-ACS over 126 orbits. We analyzed 5078 light curves, including a careful selection of 2215 cluster-member M0-M9 dwarfs. The light curves were corrected for systematic trends and inspected using several tools. No high-significance planetary candidate is detected. We compared this null detection with the most recent results from Kepler, showing that no conclusive evidence of lower planet incidence can be drawn. However, a very small photometric jitter is measured for early-M cluster members $(\$ 2 \mathrm{mmag}$ on $98 \%$ of them), which may be worth targeting in the near future with more optimized campaigns. Twelve variable stars are reported for the first time.
\end{abstract}

Key words. techniques: photometric - planetary systems - globular clusters: individual: NGC 6397

\section{Introduction}

More than seven-hundred extrasolar planets are known (exoplanet.eu database). Most of them are characterized only through radial velocities (RV) and lack any information about their "real" mass $M_{\mathrm{p}}$, because only $M_{\mathrm{p}} \sin i$ can be measured, where $i$ is the inclination of the orbit with respect to the line of sight. Their size is also unknown, which prevents us from getting any clues about their density and physical composition. Exoplanetary transits are highly complementary to $\mathrm{RV}$ techniques, providing the planetary radius $R_{\mathrm{p}}$ from the stellar radius $R_{\star}$ in a direct geometrical way (Seager 2011, p. 55). Photometric searches for transits can also go much deeper than RVs in magnitude, and can monitor thousands of stars simultaneously. The Kepler mission (Borucki et al. 2010), for instance, has demonstrated the power of this technique by discovering many planetary systems with unexpected properties. Hundreds of Kepler "candidate planets", for which confirmation and mass measurement via RVs remains infeasible, are still very useful for statistical purposes (Howard et al. 2011; Schlaufman \& Laughlin 2011).

* Based on observations with the NASA/ESA Hubble Space Telescope, obtained at the Space Telescope Science Institute, which is operated by AURA, Inc., under NASA contract NAS 5-26555.

$\star \star$ Visiting Ph.D. Student at STScI (DDRF D0001.82432 program).
Star clusters, and in particular globular clusters (GCs), offer a unique opportunity to study how the chemical and dynamical environments affect planetary formation and evolution. They are also comprised of stars that share (in most cases) the same age and chemistry, and whose radii $R_{\star}$ and masses $M_{\star}$ are reliably known on their main sequence (MS). Open clusters (OC) have been targeted for extensive transit searches (Mochejska et al. 2005; Montalto et al. 2007, 2011; Hartman et al. 2009, most notably) but only a handful of weak, unconfirmed candidates have so far been detected (Mochejska et al. 2006; Montalto et al. 2011). A global reanalysis suggests that the overall statistical significance of these campaigns is so low that it could be compatible with the planet host incidence observed in the field (van Saders \& Gaudi 2011). On the other hand, GCs are on average much richer in stars than OCs, providing a much higher statistical significance in the case of a null detection. The only GCs monitored for transits have been 47 Tucanae for both 8 days with Hubble Space Telescope (HST) WFPC2 (Gilliland et al. 2000) and 33 days from the ground (Weldrake et al. 2005); and $\omega$ Centauri with a 25-day ground-based campaign (Weldrake et al. 2008). No planetary detection has been claimed, and Gilliland et al. (2000) concluded that the planetary occurrence in 47 Tuc is smaller by a factor of ten than in field stars. The reasons that have mostly been hypothesized to explain the lack of giant planets in GCs are their metallicity and their dynamical environment. 
It has long been known that metallicity is a strong primary parameter that correlates with the fraction of stars with planets $\Phi_{\mathrm{p}}$. For giant planets, Fischer \& Valenti (2005), among others, measured an increase from the typical value $\Phi_{\mathrm{p}}^{\odot} \sim 0.03$ for stars with solar metallicity up to $\Phi_{\mathrm{p}} \sim 0.25$ for very metalrich $([\mathrm{Fe} / \mathrm{H}] \gtrsim+0.3)$ stars. For moderately metal-poor stars $(-0.5 \lesssim[\mathrm{Fe} / \mathrm{H}] \lesssim 0)$, it is still disputed whether this correlation becomes flat at values $\Phi_{\mathrm{p}} \simeq \Phi_{\mathrm{p}}^{\odot}$ (Udry \& Santos 2007; Santos et al. 2011) or $\Phi_{\mathrm{p}}$ continues to decrease exponentially towards lower metallicities (Johnson et al. 2010). As more and more lowmass planets $\left(M_{\mathrm{p}} \lesssim 30 M_{\oplus}\right)$ are being discovered, it becomes clear that their $\Phi_{\mathrm{p}}$ is much larger than that of giant planets (Lovis et al. 2009; Wittenmyer et al. 2011), probably around 20-30\%. The occurrence of low-mass planets seems to be insensitive to the host star metallicity (Udry et al. 2006), except maybe for very late-type stars (Johnson \& Apps 2009; Schlaufman \& Laughlin 2011) but the M-dwarf metallicity calibration is still too uncertain to draw conclusions.

The complex dynamical environment of a cluster is the second major concern about the survival of protoplanetary systems. Theoretical studies give different answers, but some of them imply that gravitational stripping processes are not strong enough to disrupt very short-period ( $P<5$ days) planetary systems, even in the densest regions of a typical GC (Fregeau et al. 2006; Spurzem et al. 2009). Planets do indeed exist in clusters. Giant planets have been detected around evolved stars belonging to open clusters (Lovis \& Mayor 2007; Sato et al. 2007), and around a pulsar in the globular cluster M4 (Thorsett et al. 1999). It is therefore difficult to understand why no planets have been detected in 47 Tuc. The search by Gilliland et al. (2000) was sensitive only to giant planets $\left(R_{\mathrm{p}} \gtrsim 1 R_{\text {jup }}\right)$ around stars in the upper MS, excluding late-K and M dwarfs. More data are needed to sample other regions of the parameter space.

On the other hand, it is convenient to search for transits around KM main-sequence stars because of their larger expected signal. These targets, even in the nearest GCs, are faint $(V>18)$ and extremely crowded. Space-based observations are necessary to achieve the needed signal-to-noise ratio $(\mathrm{S} / \mathrm{N})$ per time unit, and to minimize the number of false positives caused by blended photometric contaminants. The wide-field imagers mounted on HST (ACS and WFC3) are unrivaled in this respect, and their large archive of deep photometric series of GCs is already available to be exploited for transit searches. However, we show in Sect. 3 that HST time-resolved photometry is influenced by many sources of systematic errors that require a careful correction. We developed specific tools to this purpose and applied them to a test case. Other data sets (such as those for 47 Tuc) will be investigated in the near future, to test whether a more optimized campaign is worth pursuing.

In this paper, we present a search for variables and planetary transits in NGC 6397, exploiting a 126-orbit data set from the HST Advanced Camera for Surveys (ACS; GO-10424; PI:Richer). NGC 6397 is a metal-poor $([\mathrm{Fe} / \mathrm{H}] \simeq-2)$ core-collapsed globular cluster, and the second-closest known (Gratton et al. 2003; Richer et al. 2008). Our work is in some sense complementary to that presented by Stello \& Gilliland (2009), who employed the same data set to study the microvariability of highly saturated red giants. Though these data were not optimized for a transit search, its unprecedented depth allowed us to search for planets on a homogeneous sample of 2215 member M-dwarf stars down to the hydrogen-burning limit. The $\mathrm{M}$ dwarfs are considered the most promising targets to discover rocky planets in the near future (Scalo et al. 2007). They are the smallest stars $\left(R_{\star} \simeq 0.1-0.5 R_{\odot}\right)$, and therefore the transit depth for a given planet is larger by a factor of $\sim 4-100$ that that of a solar-type host star. They are also intrinsically faint $\left(L_{\star} \simeq 0.02-5 \times 10^{-5} L_{\odot}\right)$, meaning that a habitable planet would have an orbital period of only $P=10-30$ days.

\section{Observations and data reduction}

Our analysis is based on the HST data set GO-10424 (PI: Richer), whose acquisition was originally made to probe the bottom of the MS and the end of the white dwarf (WD) cooling sequence in NGC 6397 (Hansen et al. 2007; Richer et al. 2008). A single $202^{\prime \prime} \times 202^{\prime \prime}$ field, located $5^{\prime}$ from the cluster core was imaged for 126 orbits with the wide-field channel of the ACS. In each orbit, a single exposure through the $F 606 \mathrm{~W}$ filter was preceded and succeeded by two F814W images, with exposure times ranging between $584 \mathrm{~s}$ and $804 \mathrm{~s}$ (median: $704 \mathrm{~s}$ ). Overall, $252 \mathrm{~F} 814 \mathrm{~W}$ frames and $126 \mathrm{~F} 606 \mathrm{~W}$ frames were secured, for a total exposure time of $\sim 50 \mathrm{~h}$. Shorter exposures $\left(t_{\exp }=1,5\right.$, $40 \mathrm{~s}$ ) were also taken to measure the fluxes of the brightest stars, but they were not used in our study.

The dynamic range of the "deep" exposures is perfect for our purposes, as the observed luminosity function (LF) of the cluster MS members peaks at $m_{\mathrm{F} 814 \mathrm{~W}} \simeq 21$ (that is, on wellmeasured stars with $S / N \sim 200$ in the same filter; Fig. 1, histogram on the right panel). Saturation occurs around MOV spectral type at $m_{\mathrm{F} 814 \mathrm{~W}} \simeq 18.7$ and the faintest cluster members (M9V) are found at $m_{\mathrm{F} 814 \mathrm{~W}} \simeq 24(S / N \sim 20)$, such that a sample of 2000 cluster $M$ dwarfs down to the hydrogen-burning limit is available to transit search (Fig. 1, left panel).

On the other hand, these data were not optimized to search for transit events, thus two aspects of the observing setup are somewhat restrictive. First, the time coverage of the frames is discontinuous, unlike in Gilliland et al. (2000). The data for $\sim 50 \mathrm{~h}$ of integration time were spread among twenty-one noncontiguous days, spanning a twenty-eight day period. This translated into a much lower completeness for our search, especially for the longest periods ( $P>3 \mathrm{~d}$, see Sect. 5). The second reason is that each pointing was shifted within a ten-position dithering pattern $\Delta x, \Delta y$ plus a subpixel offset $\delta x, \delta y$. While dithering is usually suitable for undersampled images (Anderson \& King 2000), it prevented us from reaching the highest photometric accuracy possible for the brightest stars. For these targets, the amount of random noise is so low that the unavoidable flatfield and pixel-to-pixel residual errors are no longer negligible. A zero-point correction, discussed in the next section, was developed to suppress these systematic errors.

We carried out the data reduction on individual biascorrected and flat-fielded. flt images provided by the HST pipeline. We employed the master input list from Anderson et al. (2008), and the code described by Anderson \& King (2006) based upon the effective point spread function (ePSF) approach first developed by Anderson \& King (2000). Four tests were performed on a subset of twenty $\mathrm{F} 814 \mathrm{~W}$ frames taken at the same integer-pixel dithering position, to choose the best reduction strategy among:

1. allowing spatially-constant perturbed ePSFs as described by Anderson \& King (2006);

2. correcting the raw frames for charge transfer (in)efficiency (CTE) with the pixel-based algorithm proposed by Anderson \& Bedin (2010);

3. using both the 1) and 2) corrections;

4. using neither.

For all the measured sources, the root mean square (RMS) values of their light curve as a function of magnitude were compared. 
V. Nascimbeni et al.: An HST search for planets in the lower main sequence of the globular cluster NGC 6397
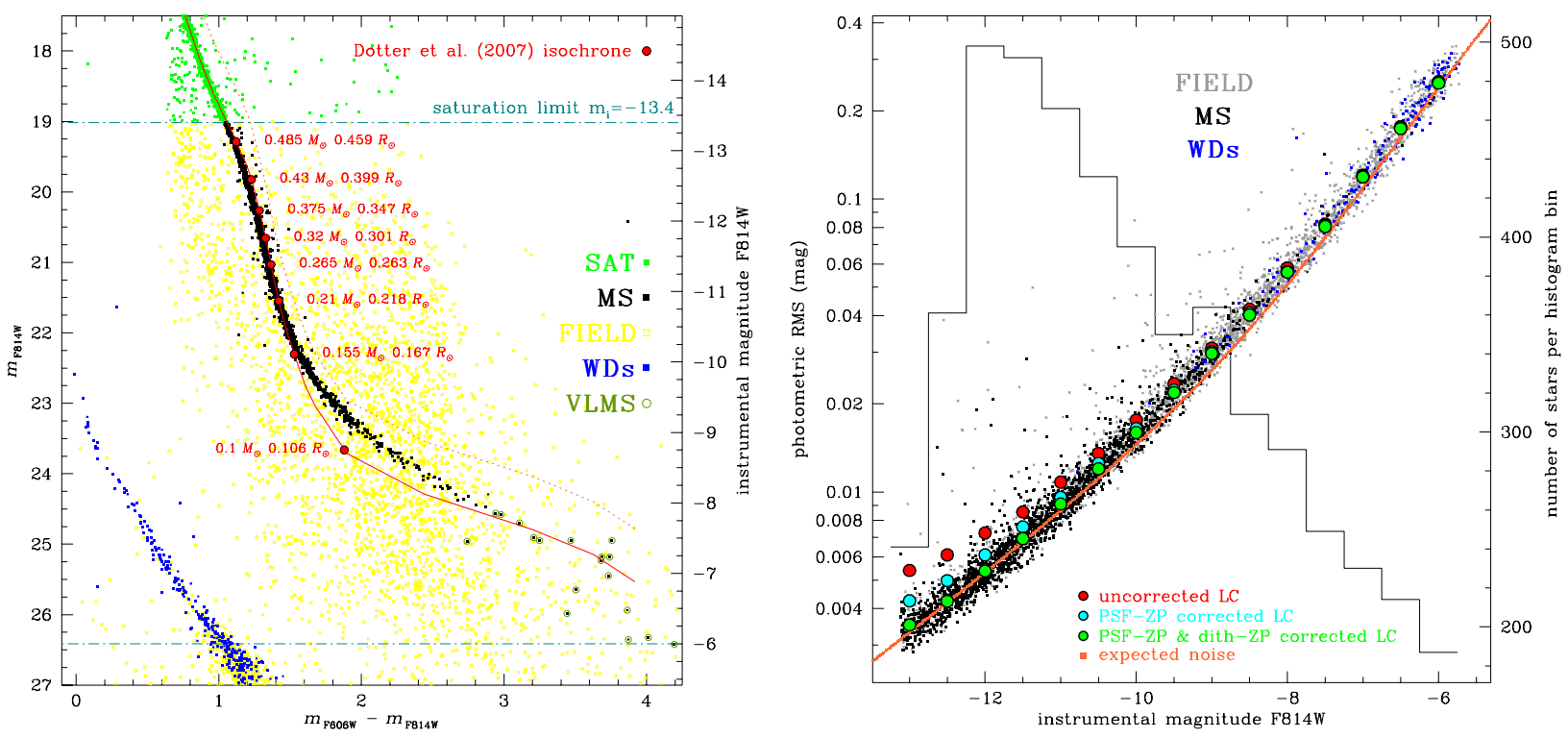

Fig. 1. Left panel. Color-magnitude diagram $\left(m_{\mathrm{F} 606 \mathrm{~W}}-m_{\mathrm{F} 814 \mathrm{~W}}, m_{\mathrm{F} 814 \mathrm{~W}}\right)$ for all the stars in the Anderson et al. (2008) master list; the 5078 sources selected in this work lie between the saturation limit at $m_{\mathrm{F} 814 \mathrm{~W}} \simeq 19$ and the faint limit $m_{\mathrm{F} 814 \mathrm{~W}} \simeq 26.5$ (dash-dot lines). The red line is the isochrone by Dotter et al. (2007) employed in Richer et al. (2008). The dotted line marks the loci occupied by equal-mass MS-MS binaries. Right panel. The rms for light curves in our sample as a function of the instrumental magnitude. Red circles: median rms averaged over 0.5 mag bins, without any correction. Cyan circles: the same, after PSF-ZP correction. Green circles: both PSF-ZP and dither-ZP corrections applied. Small points are the $\mathrm{rms}$ of the individual light curves after both corrections. The solid orange line is the expected theoretical noise level. The superimposed histogram represents the number of targets in each 0.5 mag bin (scale at the right).

Methods 1-3 above provided no detectable improvement over the fourth choice, therefore we decided not to apply any CTE or PSF correction at this stage. Actual PSFs are both spatially and temporally variable, and require a frame-to-frame a posteriori correction that is explained in Sect. 3.

Hereafter, we focus our analysis on only F814W frames for many reasons. They are much deeper than F606W images for faint, red, low MS stars, and less affected by PSF short-term variations as shown for this very same dataset by Anderson \& King (2006). The sky background is also much lower in F814W exposures. The way in which the F814W frames were sampled (at the beginning and the end of each 96-min orbit) ensures that the F606W frames are nearly useless for increasing the transit detection efficiency. In this way, we also avoided the need to correct for any tricky registration of the light curves between the two filters.

Sources beyond the saturation limit of the longest exposures $\left(m_{\mathrm{F} 814 \mathrm{~W}} \lesssim 19\right)$ and sources detected in fewer than 200 frames $\left(m_{\mathrm{F} 814 \mathrm{~W}} \gtrsim 26.5\right)$ were excluded from this study, leaving $5078 \mathrm{ob}-$ jects including cluster members, field stars, and a limited number of non-stellar objects. We evaluated the membership of each entry by performing a selection on the proper motions between our epoch and the archival ACS GO-11633 data set (PI: Rich), centered on the same field. We flagged 2430 sources as cluster members, of which 215 belong to the white dwarf (WD) sequence (see the CMD in Fig. 1, left panel). The remaining 2215 MS stars are red dwarfs ranging from M0V spectral type $\left(R_{\mathrm{p}} \simeq 0.5 R_{\odot}, M_{\mathrm{p}} \simeq 0.5 M_{\odot}\right)$ down to the hydrogen-burning limit, as confirmed by superimposing an isochrone by Dotter et al. (2007) on the CMD, as done in Richer et al. (2008).

\section{Systematic correction}

The instrumental magnitude $-2.5 \log (\mathrm{DN})$ of each star was registered to the median instrumental magnitude of stars measured in the deepest frame of the F814W series. We refer to this magnitude as $m$. Saturation occurs at $m \lesssim-13.4$. The rms $\sigma_{m}$ of our full sample of 5078 light curves was compared with the expected noise budget, as calculated by combining theoretical photon-, dark-, background- and readout noises (right panel of Fig. 1, orange line). Individual $\sigma_{m}$ were averaged whitin 0.5 mag bins by applying a clipped median (red circles in the same plot) to exclude outliers from the comparison.

On the bright side, the observed noise level on average is far higher than expected, even by $50-60 \%$ for stars with $m \lesssim-12$ (red circles, right panel of Fig. 1). We identified the source of most of this excess noise as a variation in the photometric zero point $(\mathrm{ZP})$ induced by systematic changes in the PSF shape. Long-term instability of the PSF was reported for ACS by Anderson \& King (2006). We noticed that this ZP change (hereafter called PSF-ZP) follows a well-defined pattern as a function of time and average $x, y$ position on the detector. The pattern can be mapped by evaluating for each star two diagnostic parameters that appear to be strongly correlated with the PSF-ZP shift:

1. the difference between the median magnitude $\langle m\rangle_{\text {beg }}$ measured in frames taken at the beginning of the orbit, and the median magnitude $\langle m\rangle_{\text {end }}$ measured in frames taken at the end of the orbit (left panel of Fig. 2, color-coded in the range $-0.02-0.02$ mag from black to red);

2 . the difference between the median magnitude of the star $\langle m\rangle^{7 \text { th }}$ measured in 16 consecutive frames taken during the seventh "visit" of the program $(2453451<$ JD $<245352)$, and the median magnitude $\langle m\rangle$ of its full light curve (right panel of Fig. 2, same scale).

The pattern is very similar in both cases. The first diagnostic $\langle m\rangle_{\text {beg }}-\langle m\rangle_{\text {end }}$ is probably a proxy of the real origin of the PSF-ZP systematic: a thermal/mechanical instability linked to the orbital phase. The dependence of PSF-ZP on time becomes evident when all the light curves with a high $\mathrm{S} / \mathrm{N}$ (i.e., measured 

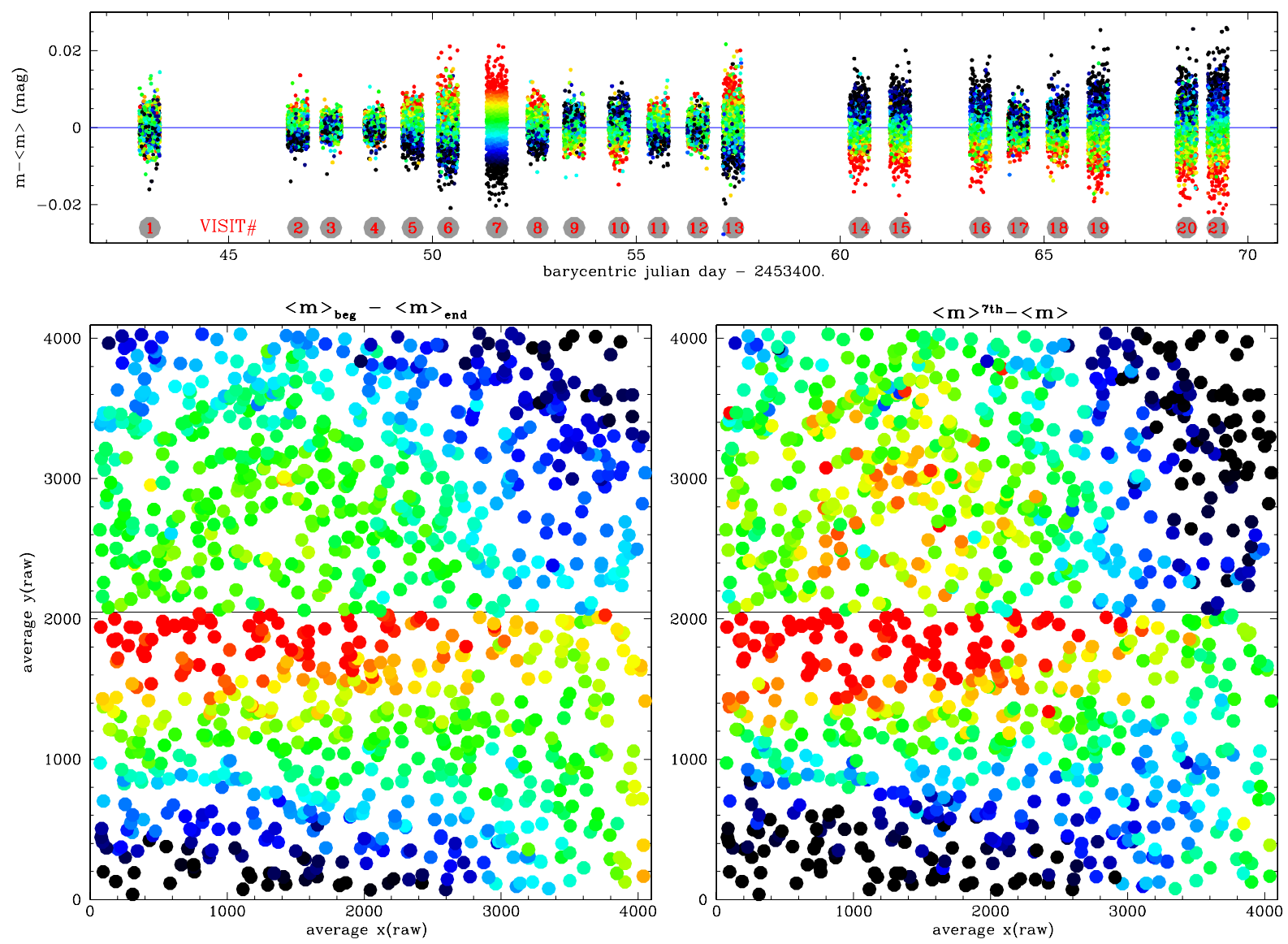

Fig. 2. Mapping the PSF-ZP shift as a function of time and position on the ACS detector, with two different diagnostics (see text). The first (left panel) is the difference between the median magnitudes $\langle m\rangle_{\text {beg }}$ and $\langle m\rangle_{\text {end }}$ measured in frames taken respectively at the beginning and the end of the orbit, while the second (right panel) is the difference between the median magnitude of the star $\langle m\rangle^{7 \text { th }}$ measured during the seventh "visit" of the program $(2453451<\mathrm{JD}<245352)$, and the median magnitude $\langle m\rangle$ of its full light curve. Top panel: all the high-S/N light curves $\left(\sigma_{m}<0.02\right)$ have been registered to their average magnitude $\langle m\rangle$. In all panels, the color scale spans the range $-0.02-0.02$ mag from black to red.

on all the 252 frames and having $\sigma_{m}<0.02$ ) are registered to their average magnitude $\langle m\rangle$, stacked in the same plot, and colorcoded as a function of $\langle m\rangle^{7 \text { th }}-\langle m\rangle$ (Fig. 2, top panel, color scale from black to red in the range -0.02 to $0.02 \mathrm{mag})$. It is clear that on average stars whose flux is overestimated during the seventh visit are also systematically underestimated in the last visits (JD > 2453460 ), and vice versa.

For a given frame and within the same chip, the PSF-ZP is a smooth function of the position on the detector. The diagnostics $\langle m\rangle^{7 \text { th }}-\langle m\rangle$ and $\langle m\rangle_{\text {beg }}-\langle m\rangle_{\text {end }}$ are too noisy when evaluated for faint stars to implement an effective correction with them. We chose instead to correct the PSF-ZP with a local approach, adapted from the differential photometry algorithms described in Nascimbeni et al. (2011). For each target star $i$ in our sample, a set of $N$ nearby reference stars $k=1, \ldots, N$ was selected with the following criteria: 1) they had to lie on the same chip as the target and be within 200 pixels of it; 2) they had to be at least 20 stars whose total flux had to exceed ten times the flux of the target; and 3) they had to be detected in at least 250 frames among 252, instead of the 200-frame limit required for a target star. When requirement 2) was not met, the search radius was increased until it was met. If on a given frame $j$ a reference star $k$ was not detected, or when its magnitude $m_{j, k}$ was more than $3 \sigma_{j, k}$ off its value averaged over the series $\left\langle m_{j, k}\right\rangle$, its magnitude was set to $\left\langle m_{j, k}\right\rangle$. A reference magnitude $m_{0, j}$ was calculated in each frame by performing the weighted mean of the magnitudes of the $N$ reference stars $m_{0, j}=\sum_{k}\left(m_{j, k} / \sigma_{j, k}^{2}\right) / \sum_{k}\left(1 / \sigma_{j, k}^{2}\right)$ (Broeg et al. 2005). The target magnitude was then normalized to $m_{0, j}$. The PSF-ZP correction was applied only when it decreased the overall rms of the target light curve. After the correction, the median rms of the light curves (right panel of Fig. 1, cyan circles) was substantially smaller. For the brightest stars $(m \lesssim-12)$, it had decreased to a level $\sim 15 \%$ above the expected noise.

Most of the remaining excess noise is due to local $\mathrm{ZP}$ changes as well as the dithering pattern employed: an integer $\Delta x, \Delta y$ shift plus a small sub-pixel offset $\delta x, \delta y$ were added to the initial pointing $x_{0}, y_{0}$ (where the units are in physical pixels). We refer to this systematic effect as dith-ZP. To each light curve, already corrected for PSF-ZP, we applied two decorrelating algorithms where

1. the median magnitude of the star $\langle m\rangle_{\Delta x, \Delta y}$ was calculated for each subset of frames sharing the same integer-pixel dither $\Delta x, \Delta y$. The magnitude $m$ of each frame in the subset $\Delta x, \Delta y$ was then registered to $\langle m\rangle_{\Delta x, \Delta y}$;

2. for each $\Delta x, \Delta y$ subset corrected by the previous step, we considered the magnitude $m_{j}$ in each frame $j$ as a function of the sub-pixel shift $\delta x_{j}, \delta y_{j}$. An ordinary linear least squares fitting was carried out to find the coefficients $c_{0}, c_{x}$, and $c_{y}$ such that

$m_{j}=c_{0}+c_{x} \delta x_{j}+c_{y} \delta y_{j}$ 
Once the best-fit value $m_{j}^{\prime}=c_{0}+c_{x} \delta x_{j}+c_{y} \delta y_{j}$ had been evaluated for each frame, the corrected light curves were extracted and normalized to zero by evaluating $m_{j}^{\prime}-m_{j}$.

Both steps 1) and 2) were applied only when the rms had decreased. The resulting median rms of the PSF-ZP + dith-ZP correction is plotted in the right panel of Fig. 1 as green circles. The small black points in the same plot show the rms of each single light curve of cluster members after our PSF-ZP and dith-ZP corrections have been applied. Their noise level approaches the theoretical limit in every magnitude bin, demonstrating that the algorithms we applied were effective.

\section{Light curve analysis}

\subsection{Search for transit-like events}

We searched for transits in the full set of 5078 light curves (that is, including field stars) by applying the box-fitting least-square algorithm (BLS, Kovács et al. 2002). For each curve, BLS was applied to search for periodic dips of duration $\Delta$ and depth $\delta$ with 10000 trial periods between 0.2 and 14 days. The relative transit duration $q=\Delta / P$ was constrained to the values possible for planetary transits around low-MS stars $\left(R_{\star}=0.08-1.4 R_{\odot}\right)$.

For each star, three detection diagnostics were calculated: the signal residue (SR), the signal detection efficiency (SDE) associated with the maximum peak in the BLS periodogram (Kovács et al. 2002), and the detection $\mathrm{S} / \mathrm{N}$ defined as

$S / N_{\mathrm{BLS}}=\frac{\delta}{\sigma} \cdot \sqrt{n_{\mathrm{t}}}$

where $\sigma$ is the (unbinned) photometric noise and $n_{\mathrm{t}}$ the number of data points sampled during transits.

Other more sophisticated detection diagnostics, such as the "signal-to-pink" S/N (Pont et al. 2006; Hartman et al. 2008), are robust if correlated noise $\sigma_{\text {red }}$ ("red noise", Pont et al. 2006) is present. However, they require knowledge of $\sigma_{\text {red }}$ on timescales close to $\Delta$. This is difficult to evaluate in our data, as transits are expected to be undersampled by the observing cadence. Transits of a $P \sim 3 \mathrm{~d}$ planet around a M4V star are expected to last $R_{\star} P /(\pi a) \sim 60 \mathrm{~min}$ at most, and only $\sim 30-40 \mathrm{~min}$ for later types, while images are sampled every 32-64 min. However, the amount of red noise here is very low, as demonstrated by the similarity of the measured rms to the theoretical one. Therefore, we decided to employ both $\mathrm{S} / \mathrm{N}_{\mathrm{BLS}}$ and SDE as detection diagnostics.

To set a reliable detection criterium, 2215 light curves were simulated, that had the same sampling times $t_{i}$ and noise level as the real M-dwarfs. A synthetic transit (following the analytical model of Mandel \& Agol 2002) of a $1 R_{\text {jup }}$ planet was injected into each curve, with a random uniform distribution in $P$ and $\sin i$ where $P$ was bounded to the range $1-5 \mathrm{~d}$, while $\sin i$ was constrained to allow transits. The process was iterated 200 times for a total of 450000 injections. We then tried to recover the transits with BLS, by setting the same parameters used for the real search. A planet was defined as "recovered" if at least two transits had been sampled, and if the estimated orbital period (or a low-order harmonics: $2: 1,3: 1,3: 2$ ) matched the injected one. The distributions of the "injected" and "recovered" transits in the $\mathrm{SDE}$ vs. $\mathrm{S} / \mathrm{N}_{\mathrm{BLS}}$ plane are plotted as black and red points in the upper left panel of Fig. 3. By dividing the parameter space into cells and evaluating the fraction of "recovered" over "injected" transits (Fig. 3, lower left panel), we obtained an estimate of the expected fraction $f$ of real transits successfully detected ("true positives"). We defined detection criteria that guarantee a fraction of false positives that is smaller than $10 \%$ (that is, $f \geq 90 \%$ ) where

$\left\{\begin{array}{l}\mathrm{SDE} \geq 5.25 \\ S / N_{\mathrm{BLS}} \geq 65-9 \cdot \mathrm{SDE} \\ S / N_{\mathrm{BLS}} \geq 7\end{array}\right.$

as indicated by the blue line in Fig. 3. With this choice, the fraction of false negatives (that is, real planets discarded by selection criteria) is about 55\%, and gets larger for planets smaller than Jupiter. This is unavoidable if one wishes to keep the fraction of false positives as low as possible.

The position of all the real sources in the $\left(\mathrm{SDE}, \mathrm{SN}_{\mathrm{BLS}}\right)$ plane is shown in the upper-right panel of Fig. 3. Only four stars among the full sample meet the criteria set in Eq. (3) or get very close to the threshold. These "borderline" targets (ID\#0269, 1961, 5936, and 7637) were inspected and cross-checked individually.

ID\#269 light curve is crippled by a CCD bad pixel falling just under the star in one of the dithering positions.

ID\#1961 is contaminated by brighter surrounding stars, and its flux drops off significantly in one fourth of the images. The BLS signal is probably spurious.

ID\#5936 seems accurately measured by the reduction pipeline, though it lies extremely close to a saturated star. Its light curve should be treated with caution. The parameters of the detected signal $(P \sim 2.1 \mathrm{~d}, \delta=0.08 \mathrm{mag}, q=0.025)$ would be compatible with a $\sim 1 R_{\text {jup }}$ transiting body with zero impact parameter, or with a grazing eclipsing binary. We classified ID\#5936 as a cluster M-dwarf member by analyzing its proper motion (with $M_{\star}=0.34 M_{\odot}, R_{\star}=0.32 R_{\odot}$ from its color), though its position in the CMD diagram is offset from the MS by $1.4 \mathrm{mag}$ in $m_{\mathrm{F} 814 \mathrm{~W}}$ and $0.2 \mathrm{mag}$ in color (Fig. 3, lower right panel). This cannot be due to binarity alone, and maybe the presence of a bright contaminant or the departure from the normal evolution of the companion play a role. There is also a non-negligible probability that ID\#5936 is a field star having a proper motion compatible with the common motion of the cluster. It it worth noting that at least one data point with a similar decrease in brightness $(0.08 \mathrm{mag}$, that is $\sim 4 \sigma)$ fell outside the expected transit windows fitted by BLS, and that the estimated duration $(\Delta=76 \mathrm{~min})$ is way larger than expected. We do not consider ID\#5936 to be a convincing planetary candidate. Its coordinates are listed in Table 1 for possible further studies. ID\#7636 was rejected because it fell over a bad column on frames corresponding to "transits".

In summary, we did not detect transits in our light curves, at least with an acceptable degree of statistical significance. We discuss the significance of this null detection in Sect. 5.

\subsection{Search for variable stars}

We performed a search for variable stars in our full database of 5078 light curves corrected for systematic errors. First, the coefficient of spectral correlation (Ferraz-Mello 1981) was calculated for each light curve. Following the method described in de Marchi et al. (2007, 2010), we obtained a sample of 13 suspected variable stars (Table 1). All these candidates, based on their proper motions and position in the CMD, were identified as field stars with high confidence. To classify these objects, a least squares iterative sine-wave search was applied (Vaníček 1971).

Most of our candidates show a single harmonic sinusoidal shape and short periods $(P \leq 9 \mathrm{~d})$, namely ID\#830, ID\#7523, 

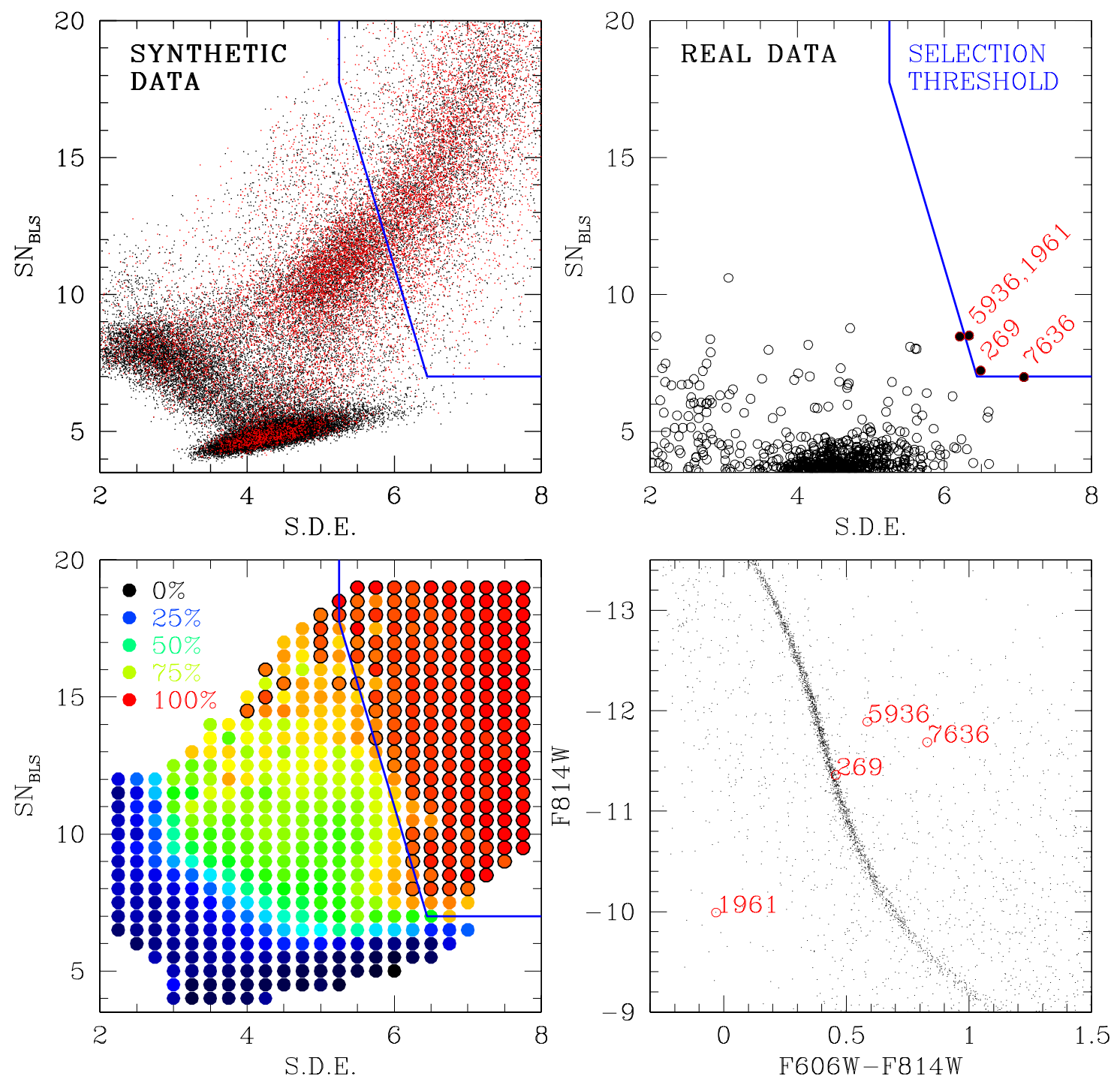

Fig. 3. Upper left: distribution of $\mathrm{SDE}$ and $\mathrm{SN}_{\mathrm{BLS}}$ from the BLS analysis of 450000 artificially injected transits in synthetic light curves. "Recovered" transits are plotted as red dots (see text). Lower left: same as above, where the parameter space has been divided into cells and color-coded as a function of the fraction $f$ of transits successfully recovered. Cells with $f>90 \%$ (that is, with an incidence of false positives smaller than 10\%) are highlighted with a black border. The blue line corresponds to the threshold defined in Eq. (3). Upper right: distribution of $\mathrm{SDE}$ and $\mathrm{SN}_{\mathrm{BLS}}$ from the BLS analysis of the full sample of 5078 real light curves. Four low-significance candidates are labeled. Lower right: location of the four low-significance candidates on the CMD.

ID\#2178, ID\#5600, and ID\#4957. Without other elements, it was impossible to derive an unambiguous classification for these variables. We suspect that these stars are most probably field BY Draconis variables, i.e. spotted and rotating KM dwarfs. This tentative classification is supported by their very red colors $\left(m_{\mathrm{F} 606 \mathrm{~W}}-m_{\mathrm{F} 814 \mathrm{~W}}=1.27-2.65\right)$.

For four stars, a good best-fit can be obtained using two harmonics. The second harmonic in the light curves of ID\#3428, ID\#1383, ID\#4430, and ID\#270 could indicate the presence of spots on the surfaces and confirm the BY Dra-type classification, while in the light curve ID\#6119 the second harmonic reveals the profile of a W UMa contact eclipsing binary system. Two stars (ID\#2086 and ID\#258) show clearly orbit-to-orbit variability but the time coverage was too short to infer reliable values for their periods: we classified them as generic "long period variables". Finally, the fluctuations in the light curve ID\#1882 are too small to allow us to confirm its nature as a variable star, hence we discarded it from our analysis. The summary classification of the entire sample is reported in Table 1, along with the best candidate transit found by BLS and discussed in Sect. 4.1.

\section{Completeness and significance}

The significance of our null detection of transits was assessed by considering only the 2215 cluster-member $M$ dwarfs for which we derived reliable estimates of $R_{\star}$ and $M_{\star}$ from their position in the CMD.

For a planet of given radius $R_{\mathrm{p}}$ and orbital period $P$, the number of expected planet detections is given by

$N_{\mathrm{p}}=N_{\star} \iint\left(\Phi_{\mathrm{p}}\left(P, R_{\mathrm{p}}\right) \cdot \Phi_{\mathrm{geo}} \cdot \Phi_{\mathrm{det}}\right) \mathrm{d} P \mathrm{~d} R_{\mathrm{p}}$,

where $\Phi_{\mathrm{p}}$ is the fraction of stars with a planet, $\Phi_{\text {geo }}$ is the geometric a priori probability for that system to be aligned such that a transit occurs, $\Phi_{\text {det }}$ is the probability for that transit to be 

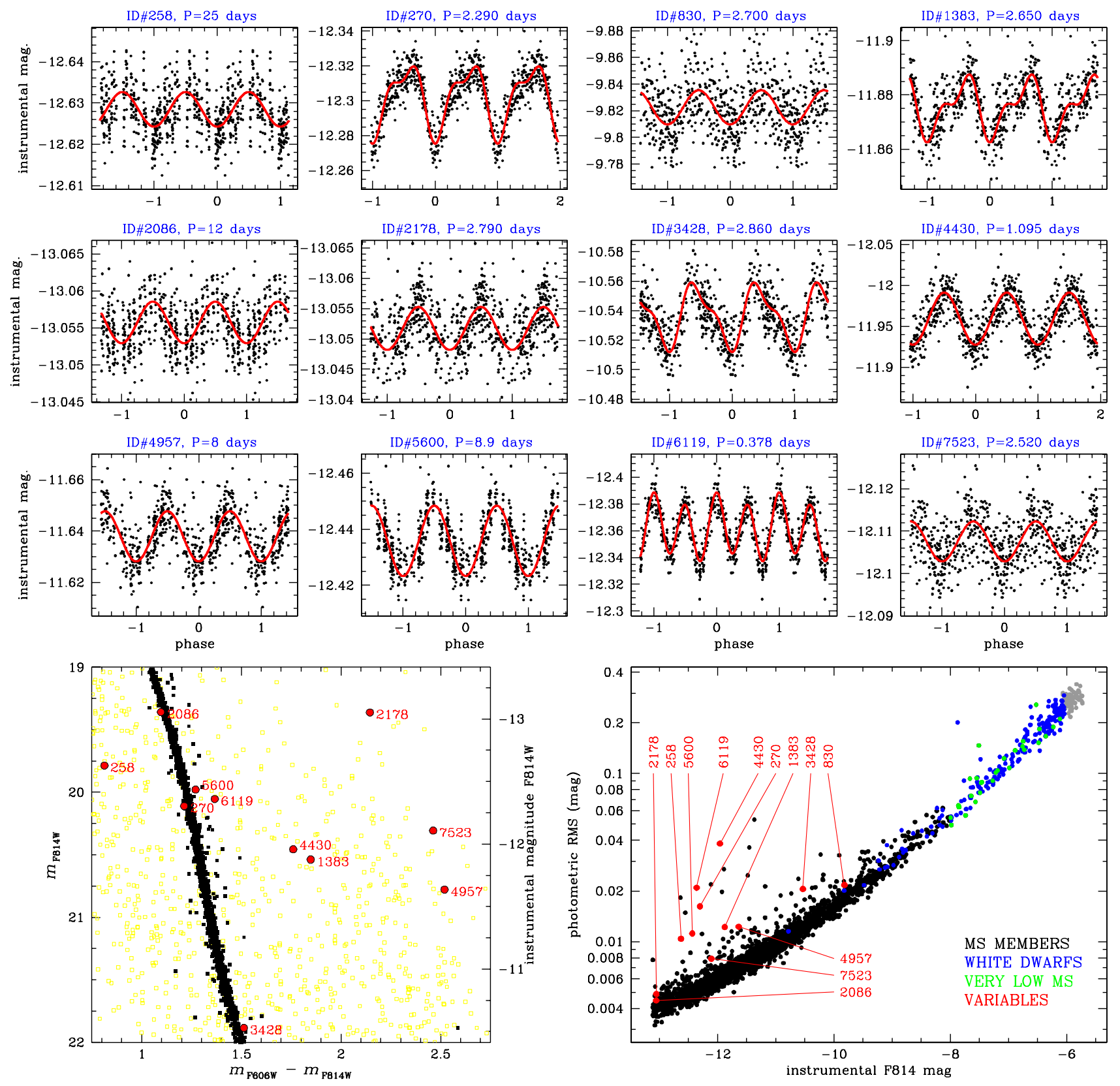

Fig. 4. Top panels: light curves of the variable stars found (first twelve entries in Table 1), folded around the best-fit period. Bottom left panel: position of the variables (red IDs) in the $\left(m_{\mathrm{F} 606 \mathrm{~W}}-m_{\mathrm{F} 814 \mathrm{~W}}, m_{\mathrm{F} 814 \mathrm{~W}}\right)$ color-magnitude diagram. Bottom right panel: photometric rms of the variables (red IDs) compared with all the analyzed light curves.

detected by our pipeline, and $N_{\star}$ is the number of target stars. As in our case we found that $N_{\mathrm{p}}=0$, we wished to estimate an upper limit to $\Phi_{\mathrm{p}}$, at least in the $\left(P, R_{\mathrm{p}}\right)$ range for which the efficiency of our search $\Phi_{\text {det }}$ is not negligible. $\Phi_{\text {det }}$ is expected to depend on the transit depth $\left(R_{\mathrm{p}} / R_{\star}\right)^{2}$, the duration $\Delta$, and the orbital period $P$.

For simplicity, our analysis was limited to two values of planetary radii: "Jupiter" planets $\left(1 R_{\text {jup }}\right)$ and "Neptune" planets $\left(0.338 R_{\text {jup }}\right)$. To estimate $\Phi_{\text {det }}$, we ran simulations in a way similar to what has been done to set the detection threshold (Sect. 4.1). In each set of 2215 simulated light curves, a synthetic transit was injected into each curve, with random uniform distributions of both $P$ and $\sin i(1 \leq P \leq 5 \mathrm{~d}, \sin i$ was constrained to allow transits). The process was iterated 200 times, for a total of 450000 injections. We then tried to recover transits with
BLS, by setting the same parameters and detection criterium defined in Eq. (3) and adopted for the real search. To derive $\Phi_{\text {det }}$, the ratio of the detected to injected transits was evaluated for each 0.1 -day bin of period $P$. The resulting distribution is plotted as a red line in the left panels of Fig. 5, as a function of the injected orbital period $P_{\text {in }}$. For comparison, the ratio of the "recoverable" transits (i.e., with at least two transits sampled and $\left.P / P_{\text {in }}=1: 1,3: 2,2: 1\right)$ to the injected transits is plotted with a green line on the same panels. As expected, $\Phi_{\text {det }}$ is a decreasing function of $P$, with minor features at integer and semiinteger values of $P$ owing to phasing effects. We note that for "Neptunes", $\Phi_{\text {det }}$ is extremely low $(0.005-0.01)$ even for short periods $(P \sim 1-2 \mathrm{~d})$. This is a consequence of inefficient sampling, which makes the significance of neptunian transits very weak: $3-4 \sigma$ even for the most favorable case. 
Table 1. Parameters for the variable stars found.

\begin{tabular}{|c|c|c|c|c|c|c|c|c|c|c|}
\hline ID\# & $\begin{array}{c}\text { RA (2000.0) } \\
\text { h:m:s }\end{array}$ & $\begin{array}{c}\operatorname{Dec}(2000.0) \\
\text { d:m:s }\end{array}$ & $\mathrm{F} 814 \mathrm{~W}$ & $m_{\mathrm{F} 606 \mathrm{~W}}-m_{\mathrm{F} 814 \mathrm{~W}}$ & $\begin{array}{l}\text { p.m. }(\alpha) \\
\text { (pixels) }\end{array}$ & $\begin{array}{l}\text { p.m. }(\delta) \\
\text { (pixels) }\end{array}$ & $\begin{array}{c}P \\
\text { (days) }\end{array}$ & $\begin{array}{c}\Delta m \\
(\mathrm{mag})\end{array}$ & $N_{\text {harm }}$ & Notes \\
\hline 3428 & $17: 41: 06.078$ & $-53: 45: 47.62$ & 21.884 & 1.513 & -1.2192 & 0.4788 & 2.860 & 0.047 & 2 & double-wave BY Dra \\
\hline 1383 & $17: 41: 11.653$ & $-53: 45: 02.54$ & 20.538 & 1.848 & -1.3037 & 0.2494 & 2.650 & 0.025 & 2 & double-wave BY Dra \\
\hline 270 & $17: 41: 14.751$ & $-53: 45: 04.94$ & 20.110 & 1.213 & -1.0478 & 0.7856 & 2.290 & 0.044 & 2 & double-wave BY Dra \\
\hline 6119 & $17: 40: 58.818$ & $-53: 45: 41.10$ & 20.054 & 1.367 & -1.4436 & 0.5940 & 0.378 & 0.051 & 2 & W UMa \\
\hline 4430 & $17: 4$ & $-53: 44: 49.11$ & 20.456 & 1.761 & 356 & 0.4401 & 1.095 & 0.069 & 2 & B \\
\hline 830 & $17: 41: 13.183$ & $-53: 45: 37.72$ & 22.598 & 2.654 & -1.3962 & 0.6337 & 2.700 & 0.026 & 1 & B) \\
\hline 7523 & $17: 40: 55.370$ & $-53: 45: 27.03$ & 20.306 & 2.463 & 1.0304 & 0.5997 & 2.520 & 0.009 & 1 & BY Dra? \\
\hline 2178 & $17: 41: 09.636$ & $-53: 43: 17.61$ & 19.362 & 2.146 & -1.6238 & 0.5057 & 2.790 & 0.007 & 1 & BY Dra? (weak) \\
\hline 5600 & 17:41:00.192 & $-53: 42: 46.28$ & 19.979 & 1.271 & -1.5276 & 0.3267 & $\approx 8.9$ & 0.025 & 1 & BY Dra? \\
\hline 4957 & $17: 41: 01.787$ & $-53: 43: 31.37$ & 20.778 & 2.520 & -1.6460 & 0.3394 & $\approx 8$ & 0.019 & 1 & BY Dra? \\
\hline 2086 & $17: 41: 09.888$ & $-53: 43: 10.59$ & 19.359 & 1.097 & -1.5737 & 0.3106 & $\approx 12$ & 0.006 & 1 & long period \\
\hline 258 & $17: 41: 14.789$ & $-53: 44: 58.36$ & 19.786 & 0.813 & -1.7434 & 0.3614 & $\approx 25$ & 0.008 & 1 & long period \\
\hline 1882 & $17: 41: 10.379$ & $-53: 44: 52.05$ & 19.430 & 0.792 & -1.0721 & 0.7363 & 0.920 & 0.005 & 1 & spurious candidate? \\
\hline 5936 & $17: 40: 59.316$ & $-53: 44: 06.51$ & 20.524 & 1.493 & 0.0181 & 0.0055 & 2.120 & 0.080 & - & grazing binary? \\
\hline
\end{tabular}

Notes. The columns give: the ID number of the star, the right ascension $\alpha$ and declination $\delta$ at epoch 2000.0, the calibrated magnitude $m(\mathrm{~F} 814 \mathrm{~W})$ and color $m(\mathrm{~F} 814 \mathrm{~W})-m(\mathrm{~F} 606 \mathrm{~W})$, the proper motion $(\alpha, \delta)$ in ACS-WFC pixels relative to the cluster, the period found in days, the amplitude found in magnitude, and the number of harmonics employed in the fit and a tentative interpretation.
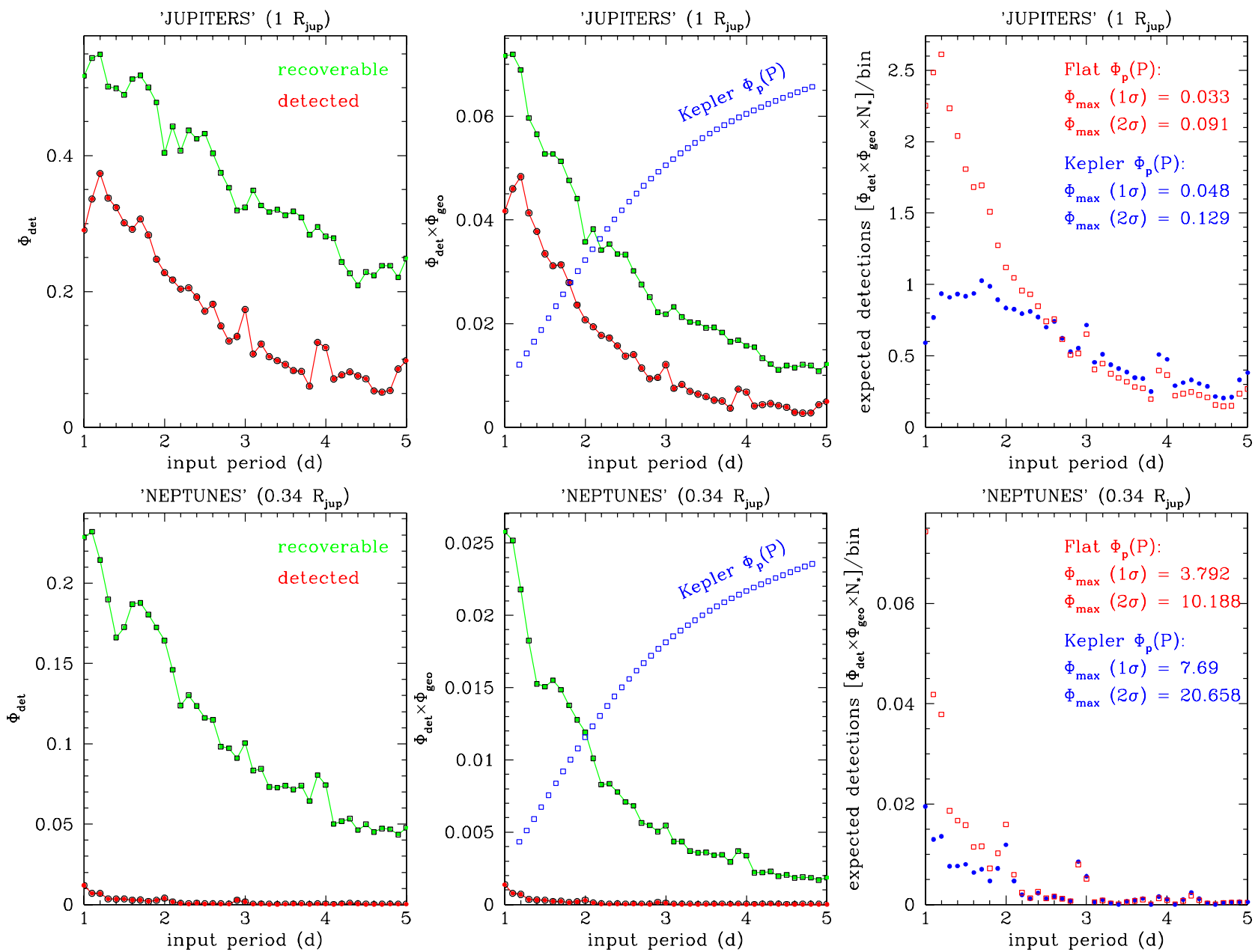

Fig. 5. Completeness tests for cluster stars based on artificially injected transits, for $1 R_{\text {jup }}$ planets (upper row) and $0.336 R_{\text {jup }}$ planets (lower row). Left panels: detection efficiency $\Phi_{\mathrm{det}}$ as a function of the input period, for planets potentially recoverable (green symbols) and for those planets effectively detected by the criterion in Eq. (3) (red symbols). Middle panels: same as above, but $\Phi_{\text {det }}$ is convolved with the geometrical probability $\Phi_{\text {geo }}$ for a planet to transit. The Howard et al. (2011) $\Phi_{\mathrm{p}}(P)$ period distribution function is plotted in blue symbols (arbitrary normalization). Right panels: number of expected transit detections per period bin, assuming one planet per star within $1<P<5 \mathrm{~d}$. $\Phi_{\mathrm{p}}(P)$ is assumed to be flat (red symbols) or as modeled by Howard et al. (2011) (blue symbols). 1- and 2- $\sigma$ upper limits for the planet occurrence $\Phi_{\mathrm{p}, \max }$ are shown. 
The geometric factor $\Phi_{\text {geo }}$ was calculated for each injected transit as $\left(R_{\mathrm{p}}+R_{\star}\right) / a$ ( $a$ is the semimajor axis), and then convolved with $\Phi_{\text {det }}$ to obtain the probability of detecting transits on a star which is known to host a planet on a random orbit, as a function of its period (Fig. 5, middle panels).

We parametrized the "planet occurrence" following the analysis of Howard et al. (2011) for the distribution of 1235 planetary candidates detected by Kepler. In this case, $\Phi_{\mathrm{p}}(P)$ was assumed to be a power law modified with an exponential cut-off at period $P_{\text {cut }}$

$$
\frac{\mathrm{d} \Phi_{\mathrm{p}}(P)}{\mathrm{d} \log P}=k \cdot P^{\beta}\left(1-\mathrm{e}^{-\left(P / P_{\text {cut }}\right)^{\gamma}}\right) .
$$

From Howard et al. (2011) we adopted the parameters: $k=$ $0.0025, \beta=0.37, P_{\text {cut }}=1.7$ days, $\gamma=4.1$ for "Jupiters" $\left(8 R_{\oplus}<R_{\mathrm{p}}<32 R_{\oplus}\right)$, and $k=0.002, \beta=0.79, P_{\text {cut }}=2.2$ days, and $\gamma=4.0$ for "Neptunes" $\left(4 R_{\oplus}<R_{\mathrm{p}}<8 R_{\oplus}\right)$. $\Phi_{\mathrm{p}}(P)$ is plotted, with an arbitrary normalization, as blue squares in the middle panels of Fig. 5.

We first normalized $\Phi_{\mathrm{p}}(P)$ by imposing $\sum_{1}^{5} \Phi_{\mathrm{p}}(P)=1$, that is assuming one planet with $1 \mathrm{~d}<P<5 \mathrm{~d}$ perstar. The total number of expected detections $N_{\exp }\left(\sum \Phi_{\mathrm{p}}=1\right)$ within each bin over the range $1<P<5 \mathrm{~d}$ is $\left(\Phi_{\text {det }} \cdot \Phi_{\text {geo }} \cdot \Phi_{\mathrm{p}}\right) N_{\star}$. By summing over the range $1<P<5 \mathrm{~d}$, we obtained $N_{\exp }=23.8$ expected detections of "Jupiters" and 0.14 of "Neptunes" (right panels of Fig. 5, blue symbols). For a flat $\Phi_{\mathrm{p}}(P)$ distribution, $N_{\exp }$ is larger at 42.3 and 0.45 , respectively.

The upper limit $\Phi_{\mathrm{p}, \max }$ to $\sum_{1}^{5} \Phi_{\mathrm{p}}(P)$ suggested by our null detection can be evaluated by simple binomial statistics, normalizing $\Phi_{\mathrm{p}}$ in order to get a $68.27 \%(1 \sigma)$ or $95.44 \%(2 \sigma)$ probability of zero detections. We estimated for Jupiters that $\Phi_{\mathrm{p}, \max }(1 \sigma)=$ $4.8 \%$ and $\Phi_{\mathrm{p}, \max }(2 \sigma)=12.9 \%$ assuming the Howard et al. (2011) $\Phi_{\mathrm{p}}$, and $\Phi_{\mathrm{p}, \max }(1 \sigma)=3.3 \%, \Phi_{\mathrm{p}, \max }(2 \sigma)=9.1 \%$ assuming a flat $\Phi_{\mathrm{p}}$. As expected, $\Phi_{\mathrm{p}, \max }$ is well above unity for the "Neptune" sample, leaving this planetary population essentially unconstrained by our data (Fig. 5, lower right panel).

\section{Discussion and conclusions}

We have performed a search for planetary transits and variability among 5078 stars imaged in one of the deepest ACS fields ever observed, which had been originally acquired to probe the bottom of the main sequence of the metal-poor globular cluster NGC 6397. The sample includes 2215 M0-M9 dwarfs of secure membership. Though these data were not optimized for such a study, this is the largest homogeneous sample of $\mathrm{M}$ dwarfs ever searched for variability.

Instrumental drifts and systematic errors caused by dithering required a careful empirical correction, described in Sect. 3. We developed and implemented algorithms that allowed us to approach the theoretical noise limit across the whole magnitude range $19 \lesssim m_{\mathrm{F} 814 \mathrm{~W}} \lesssim 26$. The brightest cluster members (M0V) were measured with an average scatter of 0.003-0.004 mag over a time span of 28 days, illustrating the power of our decorrelating techniques and the feasibility of transit searches in the low main sequence of GCs.

We found no valid planetary transit above the significance threshold that we set from simulations. Considering only cluster stars, whose physical parameters can be reliabily infered, this null detection sets an upper limit to the fraction of stars hosting a $P<5 \mathrm{~d}$ Jupiter-sized planet to about $\Phi_{\mathrm{p}}=4.8 \%$ at $1-\sigma$ confidence and $12.9 \%$ at $2-\sigma$, assuming the planetary radius distribution derived by Howard et al. (2011) from Kepler data. In other words, only 0.13 detections are expected assuming that the underlying planetary population is similar to that studied by Kepler. Most studies based on RVs also hypothesized $\Phi_{\mathrm{p}}<1 \%$ for short-period, Jupiter-sized planets around solartype stars (Marcy et al. 2005). Furthermore, $\Phi_{p}$ is expected to be a very steep function of the stellar metallicity (Fischer \& Valenti 2005). Therefore, we are unable to make any firm conclusion about the occurrence of giant planets in NGC 6397.

As demonstrated in Sect. 4.1, our data set is not sensitive enough to Neptune-sized planets to draw any conclusion about their occurrence, though a much higher $\Phi_{\mathrm{p}}$ is expected for M dwarfs by Howard et al. (2011) and Lovis et al. (2009), among others. This was due to an inefficient sampling of the time series available from archive material, which translated into poor phase coverage and severe undersampling of transit-like events, whose duration is expected to be of the same order as the effective cadence.

Twelve new variable stars have been identified in the NGC 6397 field (Table 1). Most of these can be classified as BY Draconis variables, that is, spotted rotating KM dwarfs. Interestingly, no variable has been detected among the 2430 cluster members. Hundreds of member early-M dwarfs $\left(m_{\mathrm{F} 814 \mathrm{~W}}<21\right.$, Fig. 1$)$ follow the expected noise on timescales of up to 28 days, though they were measured with a 0.003-0.006 mag precision. The lack of eclipsing binaries is unsurprising. The number of expected detections can be estimated by scaling down the number of EBs detected by Albrow et al. (2001) for 47 Tuc. If one takes into account the smaller fraction of binaries in NGC $6397(<3 \%$, even at radii smaller than the half-mass radius, Milone et al. 2012) and the smaller number of targets (2215 vs. 46422), we would expect much less than one $\mathrm{EB}$. On the other hand, the lack of BY Dra variables is more puzzling, as one would expect 4-5 such detections, considering the number of monitored cluster stars in this paper. We note that our stars are cooler than the Albrow et al. (2001) sample, and we should expect longer photometric periods. Our search is insensitive to periods $\gtrsim 28$ days, so this could be a possible explanation.

We evaluated an upper limit to the photometric jitter $\sigma_{\text {jit }}$ of the brightest members (M0V) by subtracting the contribution of the expected noise $\sigma_{\exp }$ to the measured scatter $\sigma_{\text {obs }}$ (that is, assuming $\sigma_{\text {jit }}^{2}=\sigma_{\text {obs }}^{2}-\sigma_{\text {exp }}^{2}$ ). The fraction of stars $f$ with $\sigma_{\text {jit }}>2$ mmag is $f \lesssim 2 \%$. This value should be compared with the results found by Ciardi et al. (2011) examining the first quarter of Kepler photometry on 2182 field M dwarfs: these data cover an interval of 33 days with an average cadence of $30 \mathrm{~min}$, which is quite similar to our cadence and time scale. They found a fraction $f \simeq 20 \%$ of stars with $\sigma>2 \mathrm{mmag}$, that is at least an order of magnitude larger fraction than that we measured in NGC 6397. The low MS of this cluster is extremely stable and therefore worth targeting using more optimized observations. The James Webb Space Telescope NearInfrared Camera (NIRCam), for instance, would be able to probe the bottom of the MS of NGC $6397\left(m_{\mathrm{F} 814 \mathrm{~W}} \sim 24\right)$ with a photometric precision better than $0.01 \mathrm{mag}$ in a single $600 \mathrm{~s}$ exposure, without any of the coverage/sampling issues mentioned above.

Most of the analysis techniques presented in this paper can also be applied (with little or no modification) to other existing ACS/WFC3 time series of rich stellar fields. This is the case for the metal-rich globular cluster 47 Tucanae, which has been imaged with ACS and WFC3 over a longer time-frame. Such a search for transits will allow us to complement the results of Gilliland et al. (2000) in a different range of spectral types and planetary masses. 
Acknowledgements. This work was partially supported by PRIN INAF 2008 "Environmental effects in the formation and evolution of extrasolar planetary system". V.N. acknowledges support by STScI grant DDRF D0001.82432. R.M.R. acknowledges support from grant GO-11633 from the Space Telescope Science Institute, under contract to NASA. We thank Ennio Poretti for helping us to identify the variable stars. We thank Aaron Dotter for providing us with the isochrones used in Richer et al. (2008) and presented in Dotter et al. (2007). Some tasks of our data analysis have been carried out with the VARTOOLS code (Hartman et al. 2008). We thank Ron Gilliland for his useful comments and suggestions.

\section{References}

Albrow, M. D., Gilliland, R. L., Brown, T. M., et al. 2001, ApJ, 559, 1060 Anderson, J., \& Bedin, L. R. 2010, PASP, 122, 1035

Anderson, J., \& King, I. R. 2000, PASP, 112, 1360

Anderson, J., \& King, I. R. 2006, PSFs, Photometry, and Astronomy for the ACS/WFC, Tech. Rep.

Anderson, J., King, I. R., Richer, H. B., et al. 2008, AJ, 135, 2114

Borucki, W. J., Koch, D., Basri, G., et al. 2010, Science, 327, 977

Broeg, C., Fernández, M., \& Neuhäuser, R. 2005, Astron. Nachr., 326, 134

Ciardi, D. R., von Braun, K., Bryden, G., et al. 2011, AJ, 141, 108

de Marchi, F., Poretti, E., Montalto, M., et al. 2007, A\&A, 471, 515

de Marchi, F., Poretti, E., Montalto, M., Desidera, S., \& Piotto, G. 2010, A\&A, 509, A17

Dotter, A., Chaboyer, B., Jevremović, D., et al. 2007, AJ, 134, 376

Ferraz-Mello, S. 1981, AJ, 86, 619

Fischer, D. A., \& Valenti, J. 2005, ApJ, 622, 1102

Fregeau, J. M., Chatterjee, S., \& Rasio, F. A. 2006, ApJ, 640, 1086 Gilliland, R. L., Brown, T. M., Guhathakurta, P., et al. 2000, ApJ, 545, L47 Gratton, R. G., Bragaglia, A., Carretta, E., et al. 2003, A\&A, 408, 529

Hansen, B. M. S., Anderson, J., Brewer, J., et al. 2007, ApJ, 671, 380

Hartman, J. D., Gaudi, B. S., Holman, M. J., et al. 2008, ApJ, 675, 1254

Hartman, J. D., Gaudi, B. S., Holman, M. J., et al. 2009, ApJ, 695, 336
Howard, A. W., Marcy, G. W., Bryson, S. T., et al. 2011, ApJ, submitted [arXiv: 1103.2541]

Johnson, J. A., \& Apps, K. 2009, ApJ, 699, 933

Johnson, J. A., Aller, K. M., Howard, A. W., \& Crepp, J. R. 2010, PASP, 122, 905

Kovács, G., Zucker, S., \& Mazeh, T. 2002, A\&A, 391, 369

Lovis, C., \& Mayor, M. 2007, A\&A, 472, 657

Lovis, C., Mayor, M., Bouchy, F., et al. 2009, in IAU Symp., 253, 502

Mandel, K., \& Agol, E. 2002, ApJ, 580, L171

Marcy, G., Butler, R. P., Fischer, D., et al. 2005, Progress of Theoretical Physics Supplement, 158, 24

Milone, A. P., Piotto, G., Bedin, L. R., et al. 2012, A\&A, 540, A16

Mochejska, B. J., Stanek, K. Z., Sasselov, D. D., et al. 2005, AJ, 129, 2856

Mochejska, B. J., Stanek, K. Z., Sasselov, D. D., et al. 2006, AJ, 131, 1090

Montalto, M., Piotto, G., Desidera, S., et al. 2007, A\&A, 470, 1137

Montalto, M., Villanova, S., Koppenhoefer, J., et al. 2011, A\&A, 535, A39

Nascimbeni, V., Piotto, G., Bedin, L. R., \& Damasso, M. 2011, A\&A, 527, A85

Pont, F., Zucker, S., \& Queloz, D. 2006, MNRAS, 373, 231

Richer, H. B., Dotter, A., Hurley, J., et al. 2008, AJ, 135, 2141

Santos, N. C., Mayor, M., Bonfils, X., et al. 2011, A\&A, 526, A112

Sato, B., Izumiura, H., Toyota, E., et al. 2007, ApJ, 661, 527

Scalo, J., Kaltenegger, L., Segura, A. G., et al. 2007, Astrobiology, 7, 85

Schlaufman, K. C., \& Laughlin, G. 2011, ApJ, 738, 177

Seager, S. 2011, Exoplanets, ed. S. Piper

Spurzem, R., Giersz, M., Heggie, D. C., \& Lin, D. N. C. 2009, ApJ, 697, 458

Stello, D., \& Gilliland, R. L. 2009, ApJ, 700, 949

Thorsett, S. E., Arzoumanian, Z., Camilo, F., \& Lyne, A. G. 1999, ApJ, 523, 763

Udry, S., \& Santos, N. C. 2007, ARA\&A, 45, 397

Udry, S., Mayor, M., Benz, W., et al. 2006, A\&A, 447, 361

van Saders, J. L., \& Gaudi, B. S. 2011, ApJ, 729, 63

Vaníček, P. 1971, Ap\&SS, 12, 10

Weldrake, D. T. F., Sackett, P. D., Bridges, T. J., \& Freeman, K. C. 2005, ApJ, 620,1043

Weldrake, D. T. F., Sackett, P. D., \& Bridges, T. J. 2008, ApJ, 674, 1117

Wittenmyer, R. A., Tinney, C. G., Butler, R. P., et al. 2011, ApJ, submitted [arXiv: 1103.4186] 